\title{
Spatially and Temporally Resolved Distributions of Liquid in an Effervescent Spray
}

JEDELSKÝ, J.; JÍCHA, M.

Atomization and sprays

2012, vol. 22, iss. 7, pp. 603-626

ISSN: $1044-5110$

DOl: https://doi.org/10.1615/AtomizSpr.2012006055

Accepted manuscript 


\section{Spatially and Temporally Resolved Distributions}

\section{of Liquid in an Effervescent Spray}

Jan Jedelsky*, Miroslav Jicha

Faculty of Mechanical Engineering, Brno University of Technology, Technicka 2896/2, 61669 Brno, Czech Republic

\section{Abstract}

We employed Planar Laser-Induced Fluorescence (LIF) in combination with stereoscopic Particle Image Velocimetry for imaging of concentration and flux fields of liquid phase in an effervescent spray. Light heating oil (LHO) was continuously atomized with aid of pressurised air. The atomizer was operated at atomizing pressures of $0.1-0.5 \mathrm{MPa}$ and Gasto-Liquid-Ratio by mass (GLR) of $2-50 \%$. Basic spectral and time-resolved LIF characteristics of natural fluorescence of LHO, used for LIF based concentration measurements, are detailed. Instant distributions of concentration and flux were processed into time-average radial profiles and into radial profiles of normalised root-mean-square (rms) fluctuations. Time-average concentration profiles are bell shaped with maximum concentration at the spray centreline and exponential decrease with radial distance. Liquid concentration based half-angles vary with operation conditions between 7.9 and $12.2^{\circ}$. GLR causes narrowing of the spray angle while inlet pressure has an indistinctive effect. Timeaverage flux profiles resemble the concentration profiles but are narrower, with half-angles of

\footnotetext{
* corresponding author, E-mail: jedelsky@fme.vutbr.cz
} 
$4.6-6.4^{\circ}$. Radial profiles of rms fluctuations of concentration (and also flux) generally show low value near the spray axis, increase with increasing radial distance and maximum close to the spray edge. Overall values of the rms fluctuations are used as a measure of spray unsteadiness and correlated with internal two-phase flow patterns predicted by a published flow map. The spray unsteadiness is relatively low when operated in annular flow regime and increases with change to bubbly flow regime. It confirms a direct link between the spray and internal flow.

KEY WORDS: effervescent atomization, combined PIV-PLIF measurements, spray structure, liquid concentration, liquid mass flux, spray unsteadiness

\section{NOMENCLATURE}

$\begin{array}{lll}C & \text { mass concentration } & \left(\mathrm{kg} / \mathrm{m}^{3}\right) \\ G & \text { mass flux density } & \left(\mathrm{kg} / \mathrm{m}^{2} \cdot \mathrm{s}\right) \\ \text { GLR } & \text { gas-to-liquid ratio by mass } & (-) \\ I & \text { fluorescence intensity } & (\mathrm{kg} / \mathrm{s}) \\ k_{x}, k_{y} & \text { constants needed for conversion from Imperial System to SI system } \\ \dot{m} & \text { mass flux } & (-) \\ N & \text { number of images } & (\mathrm{Pa}) \\ p & \text { air gauge pressure } & (\mathrm{mm}) \\ r & \text { radial distance } & (\mathrm{mm}) \\ s & \text { pixel area } & (\mathrm{s}) \\ t & \text { time } & (\mathrm{m} / \mathrm{s}) \\ w & \text { velocity component in the } z \text { direction }\end{array}$


$X, Y \quad$ coordinates of two-phase flow map

$x, y, z$ Cartesian coordinates

$(\mathrm{mm})$

Greek symbols

$\begin{array}{lll}\mu & \text { dynamic viscosity } & \left(\mathrm{N} \cdot \mathrm{s} / \mathrm{m}^{2}\right) \\ \rho & \text { density } & \left(\mathrm{kg} / \mathrm{m}^{3}\right) \\ \sigma & \text { surface tension } & (\mathrm{N} / \mathrm{m}) \\ \tau & \text { time constant } & \text { (s) }\end{array}$

subscripts

a air

$i \quad$ index number

$g \quad$ atomizing gas (air)

$l \quad$ atomized liquid (light heating oil)

$n \quad$ an instant value normalized by its mean value

w water

superscripts

$\left(^{-}\right)^{\prime}$ root-mean-square fluctuating value

$\left(^{-}\right)$time-average value

\section{INTRODUCTION}

Effervescent atomization has been employed in various industrial applications during past two decades. For some of them, such as combustion, surface coating and powder generation, suitable spatial distribution of the atomized liquid and/or temporal stability of the spray are 
important factors. Distribution of fuel mass within the spray volume governs mixing ratios of fuel with air in reacting sprays thus affecting combustion efficiency as well as pollutant formation. The spray cone angle is crucial for piston engines as well as for stationary combustion chambers, where the spray-wall contact should be avoided. Mass flux of sprayed liquid determines spray impact, which is of special interest in spray painting, drying and chemical processes. The Liquid mass flux controls the heat release from the burned fuel or the distribution of the cooling power of a water spray in steel rolling. These applications often require a temporal stability of the above mentioned parameters. However atomization is a dynamic process generally and for example the effervescent sprays are known to be inherently unsteady (Luong and Sojka 1999).

The structure of the spray and the shape of the spray envelope has been studied several ways. The basic spray parameter - spray cone angle (SCA), which describes borders between the spray and the ambient, is often estimated from photographic (Chen and Lefebvre 1994) or video camera (Sovani et al. 2001) documentation of the spray or measured directly using a protractor (Sivathanu et al. 2010). A proper illumination and a suitable method for definition of the spray borders is required; uncertainties in photographic estimation are mainly given by guesstimated relation between light scattered by droplets and their surface or mass.

Another technique, widely used in the spray quality control, is the isokinetic patternation. This mechanical method scans the liquid mass flowing through a given cross section (mass flux). It is simple but it suffers from several limitations such as intrusiveness and long measurement time. Precise adjustment of isokinetic probes is important especially in the case of pneumatic sprays where gas flux is not negligible (McVey et al. 1987, Cohen and Rosfjord 1991).

Phase-Doppler anemometry (PDA), well established robust and non-intrusive method for particle sizing, has been also engaged for the spray patternation (Bachalo 1994). Simply 
applied, this method produces highly biased results of mass flux and despite of significant effort to correct the results (Saffman 1987, Sommerfeld and Qiu 1995, Roisman and Tropea 2001, Dullenkopf et al. 1998) it is little used for patternation. Several other laser based instruments appeared in the past 15 years. Lazaro et al. (2003) proposed a novel laser scattering patternator which interestingly combines Phase-Doppler and Mie-scattering measurements for volume flux distributions in optically dense sprays. Laser sheet imaging employing Mie-scattering is often used for the qualitative classification of the spray characteristics such as SCA, spray solidity and near-nozzle spray structure (Gadgil et al. 2011, Daviault et al. 2012).

Fluorescence based methods such as Planar Laser Induced Fluorescence (PLIF) allow for quantitative measurements of mass concentrations (Koh et al. 2003, Sankar et al. 1999). Light emitted from an ensemble of droplets due to fluorescence is proportional to the droplet volume and therefore to the liquid concentration in the inspected volume, unlike Miescattering which is proportional to the droplet surface. LIF based methods for the spray probing are thus called "optical patternation". PLIF technique combined with Particle Image Velocimetry (PIV) or other suitable method for droplet velocity measurement extends the results to liquid mass flux (Berg et al. 2003). A planar droplet sizing technique, based on combined measurement of LIF intensity and scattered light intensity of spray droplets was used by Domann and Hardalupas (2002) to quantify the unsteadiness in a spray of a pressureswirl atomizer. Rms fields of liquid volume, surface area and Sauter Mean diameter (SMD) normalized by their corresponding local mean values were used as an estimation of the spray unsteadiness. It was shown that the spray unsteadiness could be identified to a different extent in the three investigated RMS fields. Further techniques established for spray unsteadiness measurement are briefly reviewed in (Jedelsky and Jicha 2008).

Liquid distribution in effervescent sprays has been studied by several methods. Whitlow and 
Lefebvre (1993) used a mechanical patternator to probe the radial liquid distribution. Chen and Lefebvre (1994) photographically investigated SCAs produced by a plain-orifice effervescent atomizer and examined influence of liquid rheological properties over a wide range of injection and ambient pressures. Also Sovani et al. in two studies $(2001,2005)$ took spray photography for SCA estimations. Gadgil et al. (2011) used laser sheet Mie-scattering and mechanical patternation for the study of the mean and instantaneous spray structure. General findings of these works show that (1) the radial distribution of the discharged liquid results from the upstream internal two-phase flow pattern, (2) with the strong effect of the gas/liquid morphology on the near nozzle flow field and that (3) the consequent axial evolution of the spray structure is controlled by the flow turbulence in the spray cone and by a mixing with surrounding air. Several investigators focused particularly on unsteadiness in effervescent spray (Luong and Sojka 1999, Heinlein and Fritsching 2006, Liu, Duan and Zhang 2010, Liu et al. 2011). All of them estimated the unsteadiness using the ideal spray theory (Edwards and Marx 1995) applied to PDA data. Despite of the above mentioned works related to effervescent sprays a little is known about spatially and temporally resolved characteristics of liquid distribution within the effervescent spray.

In the present paper we apply PIV-PLIF technique to an effervescent spray. This nonintrusive method overcomes limitations of the mechanical patternation when applied in a spray with strong gas flux such as the necessity of isokinetic probe usage with time consuming adjustment. LIF imaging is a direct method for quantitative measurement of mass concentration (unlike Mie-scattering based laser sheet imaging) and allows for simple evaluation of spray cone angle in contrast with the photographic documentation of the spray which requires an a priori definition and suitable method for determination of the spray borders. The combination of the PLIF with stereo PIV in our application extends the ability of the PLIF system to estimate also the mass flux density which is of special interest e. g. in 
process industry.

We have used the PIV-PLIF technique for an examination of a spray generated by a simple effervescent atomizer. Temporally mean and fluctuating concentration and mass flux density images, acquired for a set of inlet pressure and GLR values, give information on spatial and temporal variation of the atomized liquid. Normalized rms fluctuations of the concentration and mass flux density were used as a measure of the spray unsteadiness and correlated with a predicted internal two-phase flow patterns. This work relates to our previous study (Jedelsky and Jicha 2008), performed on the same optically transparent atomizer, where visualized internal two-phase flow patterns were compared with the patterns predicted by a two-phase flow map and two methods were employed to estimate the effect of operation conditions on the spray unsteadiness.

\section{EXPERIMENTAL}

An effervescent atomizer was operated on a cold test bench and observed by means of stereoscopic PIV-PLIF system.

\subsection{Atomizer Description and Operation}

A simplified transparent Plexiglas version of an industrial effervescent atomizer was used for the experiments. Fig. 1 shows its geometry. This optically accessible atomizer was mainly designed to allow an internal two-phase flow visualization (Jedelsky and Jicha 2004). The liquid flows in the main channel from top, while the air enters by side channels and it is injected into the liquid, from each side, through a set of 15 holes with $1 \mathrm{~mm}$ in diameter. Internal diameter of the mixing chamber is $8 \mathrm{~mm}$ and the length, downstream of the last row of air holes, is $80 \mathrm{~mm}$. The generated mixture flows downstream and exit the atomizer through an orifice into atmosphere. The exit orifice, made of a brass plate, has a diameter of $2.5 \mathrm{~mm}$ and a length of $0.7 \mathrm{~mm}$. A conical junction with the apical angle of $90^{\circ}$ is formed 
between the orifice and the mixing tube.

The atomizer was continuously operated and studied in the vertical downward position of the main axis on a cold test bench. LHO was supplied into the atomizer from a main fuel tank by a gear pump through filters and control valves. The compressed air was delivered from the central plant through an air chamber, filters and a control valve. The air and oil supplies of the twin-fluid atomizer were controlled separately; operation conditions were adjusted by the air gauge pressure and by GLR. All other parameters such as liquid pressure, liquid and air flow rates, were dependent upon them. Since one of the goals of this study was to investigate how the spray structure depends on the atomizer operation conditions, experiments were performed at constant air gauge pressure $p=0.2 \mathrm{MPa}$ with GLR varying in range of $2-50 \%$ and at constant GLR of $5 \%$ with varying $p$ in range of $0.1-0.5 \mathrm{MPa}$. Temperatures, gauge pressure and volumetric flow rates were measured at the atomizer inlets for both the fluids. Physical properties of the LHO at room temperature are: surface tension $0.0297 \mathrm{~kg} / \mathrm{s}^{2}$, density $874 \mathrm{~kg} / \mathrm{m}^{3}$, dynamic viscosity $0.0185 \mathrm{~kg} / \mathrm{m} \cdot \mathrm{s}$. For a detailed description and diagram of the experimental facility see (Jedelsky and Jicha 2008).

\subsection{Stereoscopic PIV-PLIF System}

Planar stereoscopic PIV-LIF system from TSI Incorporated (Fig. 2) was used for measurements of the velocity and liquid concentration distributions. Double pulsed Nd:YAG SLII-10 laser (Continuum Inc.) (1-3) equipped with 4th harmonic generator (2) provides laser pulses at $265 \mathrm{~nm}$ with duration of $\sim 4 \mathrm{~ns}$. The laser beam passes through mirrors (8) and is conditioned with a light sheet generator (6) to a sheet of approximately $100 \mathrm{~mm}$ width and $2.5 \mathrm{~mm}$ thickness, which defines the measurement plane. The light sheet illuminates the spray in a cross section perpendicular to the spray axis in the distance $152 \mathrm{~mm}$ from the nozzle exit (4). 
This position of the measurement plane was chosen as a compromise value for the following reasons. This distance is often used by the other researchers ( Whitlow and Lefebvre 1993, Sutherland et al. 1997). Our PDA measurements at several axial distances from the exit orifice (50, 100, 150, and $200 \mathrm{~mm})$ (Jedelsky et al. 2009) indicate a fully developed spray at the distance of $150 \mathrm{~mm}$. Very dense spray in the near-nozzle region causes namely multiple scattering effects and significant laser light attenuation which would require PLIF corrections. Furthermore, the spray is not fully developed when too close to the nozzle orifice. Spray measurement at distances larger than $150 \mathrm{~mm}$ from the atomizer exit is of limited value for combustion applications, as the droplets would interact with the combustor flow field and evaporate. The image capture system consists of two PIVCAM 13-8 cameras (TSI Inc.) with Scheimpflug optics and $28 \mathrm{~mm}$ lenses with appropriate optical filters (5L, 5R). The CCD cameras are placed on the same side of the light sheet (back- and forward light scatter) with the full stereoscopic viewing angle of $90^{\circ}$. This angle provides sufficient accuracy for the estimation of the out-of-plane velocity component (Adrian 2011). Timing of the PIV system is controlled by a LaserPulse synchronizer (TSI Inc.) (9) in concert with an acquisition computer (7). The cameras receive a series of image pairs, each from one of the laser pulses. The double images are processed with a PIV algorithm to provide an instantaneous theecomponent vector field of droplet velocity in the plane perpendicular to the spray centreline. The same images are also used as LIF intensity field, which represents the liquid density information. LHO used for experiments is naturally fluorescent. Laser excites in the LIF active molecules of the liquid at the wavelength of $265 \mathrm{~nm}$. Molecules absorb the light and radiate it at higher wavelength. Emitted light intensity is proportional to the excited molecule volume and hence to the liquid concentration. To record only the fluorescence light a bandpass filter XF3004 (Omega Optical, Inc.) is used in front of the camera lens. The filter transmits about $70 \%$ of light within a band given by cutoff wavelengths 390 and $435 \mathrm{~nm}$. 
The 3-C PIV system was calibrated by a two-plane calibration target. For each experimental run, 256 paired images were captured and processed by a PIV processor after background subtraction according this setup: Grid Engine: Deformation Grid, Spot Mask Engine: Deformation Mask, Correlation Engine: FFT Correlator, Peak Engine: Gaussian Peak, Starting spot dimension $64 \times 64$ and final spot dimension $32 \times 32$. Spurious vectors were rejected using interactive validation employing a set of Global Standard Deviation, Local Median and Local Mean filters.

Each of the experimental runs, differing in the inlet pressure and GLR, required different time delay between laser pulses (from 10 to $40 \mu \mathrm{s}$ ) in order to minimize the out-of-plane motion of particle images between the paired captures, as well as the in-plane loss of correlation due to large particle displacement at higher velocities. Also the relatively thick light sheet of 2.5 mm was chosen to prevent the out-of-plane loss of correlation between the paired captures due to main velocity component oriented perpendicularly to the illumination plane. The images were acquired and processed using Insight 3G 9.1 (TSI Inc.) software package and further, bellow described analysis was done in MATLAB7 software.

\subsection{Fluorescence of LHO}

Natural fluorescence of LHO is utilized for PIV and LIF measurements. Knowledge of basic characteristics of the fluorescence is required for correct setup of the measurement system and verification of its application. Tests of fluorescence were done using the spectrometer FLS 920 (Edinburgh Instruments). Fig. 3 shows the emission spectra for the excitation wavelengths $266 \mathrm{~nm}$ and $355 \mathrm{~nm}$. Fluorescence maximum is $416 \mathrm{~nm}$ for both the cases. The $416 \mathrm{~nm}$ emission wavelength was used for the illustration of the variation of the emission efficiency with the excitation wavelength. The maximum fluorescence yield is seen at excitation wavelength $355 \mathrm{~nm}$. Although this wavelength is optimum, the wavelength $266 \mathrm{~nm}$ 
was chosen for PIV-LIF measurements as it enables an easier separation of the fluorescence signal from the excitation signal using optical filters.

Lifetime of the fluorescence was measured for an excitation pulse with wavelength $266 \mathrm{~nm}$ and duration of $7 \mathrm{~ns}$. Results for the emission wavelength $416 \mathrm{~nm}$ (corresponding to maximum luminescence) are seen in Fig. 4. The fluorescence decay is modelled using a twoexponential function $I=0.209+0.007 \exp \left(-t / \tau_{1}\right)+0.004 \exp \left(-t / \tau_{2}\right)$ with the time constants $\tau_{1}=(7.16 \pm 0.17) \mathrm{ns}$ and $\tau_{2}=(18.7 \pm 0.2) \mathrm{ns}$. Both the values are negligible in relation to the time scales of the PIV measurements. These results justify the usage of the natural fluorescence of LHO for both the PIV and LIF measurements. Dosing by a fluorescent dye would not probably bring any significant improvement in the measurement but it could lead to complications regarding to required uniformity of dispersion in the LHO. Signal of fluorescence was found to have linear dependence on the excitation intensity in the whole range of laser power used in the measurements with no variation in the shape of the emission spectra curve.

\subsection{Data Processing}

A set of image pairs of the LIF intensity was processed into instantaneous three-component velocity vectors of the droplets and the liquid phase concentration (mass per unit volume) in the $(x, y)$ plane, perpendicular to the spray centreline, $z$.

Instantaneous images of the liquid concentration $C_{i}(x, y)$ (Fig. 5) and the liquid mass flux density $G_{i}(x, y)$ provide a statistical information about temporally varying distributions of the liquid in the $(x, y)$ cross section. An image of liquid mass flux density in the axial direction $G_{i}(x, y)$ (the indices $l$ for liquid and $z$ for axial direction specification are omitted for conciseness) is acquired as a combination of the axial (out-of-plane) velocity component, $w_{i}(x, y)$, and the concentration image 


$$
G_{i}(x, y)=w_{i}(x, y) \cdot C_{i}(x, y)
$$

Recorded set of $N=256$ images is used to calculate the mean image representing the spatially resolved, time-average liquid mass concentration:

$$
\bar{C}(x, y)=\frac{1}{N} \sum_{i=1}^{N} C_{i}(x, y)
$$

and the same way also flux density $\bar{G}(x, y)$. Spatial distribution of rms fluctuations of the liquid mass concentration normalized by the local mean value is as follows:

$$
\overline{C_{n}^{\prime}}(x, y)=\frac{1}{\bar{C}(x, y)} \sqrt{\frac{1}{N} \sum_{i=1}^{N}\left[C_{i}(x, y)-\bar{C}(x, y)\right]^{2}}
$$

This quantity as well as normalized rms fluctuations of flux density, $\overline{G_{n}^{\prime}}$, (calculated the same way) describe an intensity of the temporal fluctuations of the liquid distribution in the spray thus serving as a spray unsteadiness measure. Instantaneous value of the liquid mass flow rate through the measurement plane is derived as:

$$
\dot{m}_{i}=\sum_{x, y}\left[G_{i}(x, y) \cdot s\right]
$$

where $s$ is a pixel area. The rms of flow rate fluctuations normalized by the mean flow rate, $\overline{\dot{m}_{n}^{\prime}}$, gives a global characterization of the spray unsteadiness. Spatially averaged value of the normalized mass flux density fluctuations:

$$
\overline{G_{n}^{\prime}}=\sum_{x, y} \overline{G_{n}^{\prime}}(x, y)
$$

shows an overall level of mass flux density fluctuations inside the measurement plane.

\section{RESULTS}

Fig. 5 documents LIF images of the spray which represent the instantaneous concentration distribution of the liquid, $C_{i}(x, y)$, in the measurement plane perpendicular to the nozzle centreline in the distance of $152 \mathrm{~mm}$ from the nozzle exit. The spray consists of small droplets 
in the case of GLR $=5 \%$ and higher (Fig. 5 left). The axial symmetry of the spray generated by the plain orifice atomizer is evident. For low GLR $\leq 2 \%$ a poor atomization occurs, ligaments of the liquid are observed even in the axial distance of $152 \mathrm{~mm}$, mainly near the spray centreline (Fig. 5 right). This effect is related to an unsteady and heterogeneous twophase mixture (plug flow) prior to discharge (Jedelsky and Jicha 2008). A similar character of the spray and the tendency with the GLR change was described in (Gadgil et al. 2011).

Representative time-average velocity field is shown in a perspective view in Fig. 6. It implies the axial spray symmetry with Gaussian-like distribution of velocity magnitude and dominant axial velocity component, $w$ (the nozzle centreline is identical to the $z$-axis, which is perpendicular to the $(x, y)$ measurement plane).

\subsection{Spatial Liquid Distributions}

Radial profiles of the liquid concentration were processed from the LIF images using the spray symmetry character and only half profiles are plotted. Fig. 7 documents radial distribution of the time-average liquid concentration, $\bar{C}$, and its cumulative value, $\bar{C}_{c}$, for air gauge pressure $p=0.2 \mathrm{MPa}$ with varying GLR. The $\bar{C}_{c}$ is calculated as

$$
\bar{C}_{c}(r)=\int_{0}^{r} \bar{C}(r) \cdot r \cdot d r / \int_{0}^{R} \bar{C}(r) \cdot r \cdot d r .
$$

where $R$ is the maximum observed diameter of the spray. The profiles are normalized by their respective maximum value to enable comparison. Logarithmic scale is used for $\bar{C}$ to emphasize relative differences amongst individual curves. Maximum $\bar{C}$ lies in the nozzle centreline. It gradually decreases up to $r \sim 10 \mathrm{~mm}$, following with an approximately exponentially decaying trend (linear trend in logarithmic scale) for low GLRs. The $\bar{C}$ values are very similar amongst individual GLR curves up to $r \sim 15 \mathrm{~mm}$. For further increase in $r$ more distinct differences in results are seen with steeper decrease for profiles with high GLR 
values. The shape of the radial $\bar{C}$ profile well corresponds to the concentration profile in an air-helium jet observed by Panchapakesan and Lumley (1993) or to a water distribution in an air-assist internally mixed spray studied by Aliabadi et al. (2011). It suggests for the same fundamental mechanisms that govern the transversal mass transport within the gas jets and different twin-fluid jets.

All cumulative radial distributions, $\bar{C}_{c}$, are typically "s" shaped with the steepest tendency within $6 \mathrm{~mm}<r<15 \mathrm{~mm}$. However the slope of the distribution varies amongst individual curves which leads to increasing difference with $r$ amongst these curves. About $90 \%$ of the liquid mass is contained in the area of $30 \mathrm{~mm}$ in radius in the case of GLR $=2 \%$. The spray cone narrows with increasing GLR; more than $90 \%$ of liquid mass is included inside the circle with $r=21 \mathrm{~mm}$ for GLR $=50 \%$.

Effect of the air gauge pressure on $\bar{C}$ and $\bar{C}_{c}$ distributions for varying inlet pressure at GLR $5 \%$ is documented in Fig. 8. Effect of the pressure on shape of the $\bar{C}$ profile is apparent mainly near the spray centreline where individual curves exhibit different slopes; declination of the $\bar{C}$ profile is steeper for lower pressures. For $r \geq 10 \mathrm{~mm}$ the curve tend to keep almost constant relative distance. $\bar{C}$ values near the centreline do not affect $\bar{C}_{c}$ distribution strongly, due to weighting by $r$, so the $\bar{C}_{c}$ does not vary largely with the air gauge pressure changes. This is in contrast with the above mentioned effect of GLR, which acts more significantly in larger radial distances.

Mass flux density of liquid in axial direction was calculated using Eq. (1) and (2). Timeaverage radial profiles of the mass flux density, $\bar{G}$, and its cumulative value, $\bar{G}_{c}$, are documented in Fig. 9 for air gauge pressure $p=0.2 \mathrm{MPa}$ with varying GLR and in Fig. 10 for varying inlet pressure at GLR 5\%.

The shape of both the $\bar{G}$ and $\bar{G}_{c}$ profiles generally resembles patterns of their corresponding 
concentration profiles, however the mass flux density profiles are significantly narrower due to weighting of the concentration by the axial velocity. The velocity profiles are similar to the $\bar{C}$ profiles; maximum $w$ is also situated in spray centreline and mainly the exponentially decaying trend with radial position is typical also for velocity. Therefore the $\bar{G}$ profiles also exhibit the linearly decaying trend in the logarithmic scale however the liquid mass flux is concentrated in a smaller region around the axis. The radial flux distribution of droplets can be well described by $\bar{G}(r) \approx \exp \left(-r^{2}\right)$ as suggested by Hetsroni and Sokolov (1971) for a two-phase turbulent jet.

Effect of GLR on $\bar{G}$ and $\bar{G}_{c}$ profiles (Fig. 9) remained the same as for $\bar{C}$ and $\bar{C}_{c}$. Differences amongst individual $\bar{G}$ profiles gradually increase with $r$; a steeper decay is observed for higher GLR values. It is well reflected in $\bar{G}_{c}$ profiles that suggest for narrowing of spray cone with GLR increase. More than $90 \%$ of liquid flows through the area with $r<$ $17 \mathrm{~mm}$ for GLR $=2 \%$. The area decreases with the GLR increase and it is $12 \mathrm{~mm}$ for GLR $=$ $50 \%$.

Effect of pressure on $\bar{G}$ and $\bar{G}_{c}$ profiles (Fig. 10) is not as distinctive. The $\bar{G}$ profiles are influenced already in the first $10 \mathrm{~mm}$ from centreline following with an indistinct tendency. $\bar{G}_{c}$ profiles are very similar one to another.

\subsection{Temporal Fluctuations of the Spray}

Intensity of the temporal fluctuations of the spray is characterised with rms fluctuations of the liquid mass concentration normalized by its local mean value, $\overline{C_{n}^{\prime}}$, calculated according Eq. (3) and with normalized rms fluctuations of the flux density, $\overline{G_{n}^{\prime}}$, calculated the same way. Radial $\overline{C_{n}^{\prime}}$ profiles (Fig. 11 and 12) generally show low values near the spray centreline and 
an increase with $r$ until reaching a maximum at $r=30-40 \mathrm{~mm}$ from which $\overline{C_{n}^{\prime}}$ decreases up to the spray edge. The influence of GLR (Fig. 11) is strong in the entire radial profile; the fluctuations are low at high GLR regimes and gradually increase with GLR decrease. Effect of $p$ is not as significant however moderate and consistent decrease with increasing $p$ can be resolved from profiles in Fig. 12.

Shape of radial $\overline{G_{n}^{\prime}}$ profiles (Fig. 13 and 14) as well as their variation with $p$ and GLR generally corresponds to the $\overline{C_{n}^{\prime}}$ patterns. The $\overline{G_{n}^{\prime}}$ maximum is placed in the space of $r=20-$ $30 \mathrm{~mm}$. The level of the fluctuations tends to increase with GLR decrease. This trend is the most distinct for $20<r<40 \mathrm{~mm}$, however in the case of GLR $=2 \%$ also a growth of fluctuations near the spray centreline is obvious.

Effect of $p$ (Fig. 14) is not as evident; all the regimes show similar shapes and levels of fluctuations with the exception of the results at $0.1 \mathrm{MPa}$, where the fluctuations significantly increased for $r<20 \mathrm{~mm}$.

\section{DISCUSSION}

Table 1 documents half-angles of temporally averaged concentration corresponding to 50, 75 and $90 \%$ of the contained mass at the inspected axial distance of $152 \mathrm{~mm}$. Results are derived from radial $\bar{C}_{c}$ profiles. The data shows a very good consistency amongst individual percentages; for example a narrow 50\% angle in the case of high GLR implies for narrow $75 \%$ or $90 \%$ angles and vice versa wide $50 \%$ angles at low GLR case result in large $75 \%$ or $90 \%$ angles. The half-angle shows a monotonic, almost linearly decreasing tendency with GLR. Effect of the pressure is indistinctive and ambiguous.

The $90 \%$ half-angle values roughly correspond to values found in (Chen and Lefebvre 1994, Sovani et al. 2005), where photographic observation was used. Chen and Lefebvre (1994) found the cone angles to widen with the atomizing air injection pressure and widen slightly 
also with GLR up to GLR $\sim 6 \%$ and then narrow for larger GLRs. Sovani et al. (2005) reported the spray cone half-angles independent of GLR or the injection pressure for GLR $=1$ $-9 \%$ and $p=12-30 \mathrm{MPa}$.

The two phase mixture after discharge flows with a dominant axial velocity component. The radial velocity, which affects the cone angle, depends on the character of the internal twophase flow and the character of the discharge. The bubbly flow at low GLR (see Fig. 15 bellow) results in bubble bursting downstream the exit orifice as reported by Chen and Lefebvre (1994) and visualised by Gadgil and Raghunandan (2011). Air, expanding during the bubble ruptures, causes the liquid to disperse in all directions. The annular flow at higher GLR, or dispersed flow at even higher GLR, lead to a formation of axially oriented ligaments of liquid (Santangelo and Sojka 1995) to which the high-speed air acts mainly in axial direction. Internal two-phase flow regime is therefore responsible for the spray narrowing with GLR.

Variation in the air inlet pressure in the range $0.1-0.5 \mathrm{MPa}$ leads to only small change in the position in the two-phase flow diagram (Fig. 15) so the two-phase flow regime should not affect the spray angle significantly with the pressure change. The pressure drop however affects the character of the discharge from the exit orifice. An increase in the pressure drop leads to an increase in the discharge velocity and the flow choking at higher inlet pressures (Leung and Epstein 1990). Radial profiles of velocity tend to narrow with pressure increase. Turbulence intensity inside the two-phase jet closely relates to Reynolds number and thus to the discharge velocity. Turbulence is responsible for a transverse movement of the mass within the spray and it therefore homogenizes the mixture and widens the spray angle. Although the $\bar{C}$ profiles (Fig. 8) vary with the inlet pressure, the final effect of the inlet pressure on the concentration angle (Table 1) is weak due to contrarily acting factors of the turbulence mixing and velocity profiles. 
Table 2 documents half-angles of temporally averaged fluxes that correspond to 50, 75 and $90 \%$ of the total mass flow at the inspected axial distance of $152 \mathrm{~mm}$. Results are derived from radial $\bar{G}_{c}$ profiles (Fig. 9 and 10). Practically linear relationship between the $\bar{C}$ and $\bar{G}$ half-angles is seen when comparing corresponding values in Table 1 and 2 . The $\bar{G}$ halfangles are of about one half of the $\bar{C}$ half-angles due to the effect of spatial velocity distribution. Droplets flow with very high axial velocity near the spray centreline and slower at higher radial positions so most of the flowing mass is concentrated in the spray core. Effect of the pressure and GLR is similar to the behaviour of $\bar{C}$ half-angles.

Temporal fluctuations of the liquid concentration and mass flux in effervescent sprays are generally related to two factors. The gas-liquid mixture inside the mixing chamber is not ideally homogeneous. Gas bubbles, typically several $\mathrm{mm}$ in diameter, are formed at low GLR (Huang et al. 2008) and even plug or slug flow (Catlin and Swithenbank 2001) can appear for specific operation conditions. This gas-liquid distribution naturally leads to a discharge with temporally varying GLR and finally to the unsteady spray (Jedelsky and Jicha 2008, Gadgil and Raghunandan 2011). Ligaments of liquid are gradually atomized by shear into small droplets however the original gas-liquid inhomogeneity remains in the form of droplet clouds. The spray at the annular flow regime benefits from a less fluctuating GLR during discharge and it is thus more steady. The second factor of the spray fluctuations is related to droplet dynamics. Droplets are exposed to a high-speed turbulent air jet in the spray core and to a slow surrounding air flow near the spray edge. Small droplets are trapped in the vortical flow while large droplets resist; it leads to the size dependent clustering or to the dispersion (Aliabadi et al. 2011, Jakubik et al. 2006).

Combination of these factors lead to the specific radial distribution of temporal fluctuations of the spray expressed with $\overline{C_{n}^{\prime}}$ in Fig. 11 and 12 and with $\overline{G_{n}^{\prime}}$ in Fig. 13 and 14. Such a specific pattern of the unsteadiness with low centreline values and maximum near spray edge was also 
documented in papers (Luong and Sojka 1999, Heinlein and Fritsching 2006). We observed the same shape of the radial profile of spray unsteadiness in our earlier work (Jedelsky and Jicha 2008) where PDA was used with the evaluation method of Edwards and Marx (1995) on the same atomizer as the one used here. Note that in that work the unsteadiness maximum was found at $r=40 \mathrm{~mm}(\mathrm{GLR}=2.6 \%, p=0.2 \mathrm{MPa})$ and here for similar regime $(\mathrm{GLR}=2 \%, p=$ $0.2 \mathrm{MPa}$ ) we register the $\overline{C_{n}^{\prime}}$ maximum at $\sim 36 \mathrm{~mm}$ and maximum $\overline{G_{n}^{\prime}}$ at $\sim 27 \mathrm{~mm}$.

Overall values of $\overline{G_{n}^{\prime}}$ and $\overline{\dot{m}_{n}^{\prime}}$, calculated according Eq. (5) were visualized in the modified Baker's map for vertical downward flow with transformed coordinates (Process Associates of America, 2003) to allow simple correlation of the spray unsteadiness with predicted twophase flow pattern in the mixing chamber of the atomizer (Fig. 15 and 16). Lines drown in the diagram distinguish between different flow regimes. Operation point of the atomizer (its $X$ and $Y$ coordinates) depends on the gas and liquid flow rates and physical properties of these fluids as

$$
X=k_{x} \cdot G L R \cdot \frac{\sigma_{w}}{\sigma_{l}}\left(\frac{\rho_{g}}{\rho_{a}}\right)^{1 / 2}\left(\frac{\rho_{l}}{\rho_{w}}\right)^{1 / 6}\left(\frac{\mu_{l}}{\mu_{w}}\right)^{1 / 3}
$$

and

$$
Y=k_{y} \dot{m}_{g}\left(\frac{\rho_{a}}{\rho_{g}} \cdot \frac{\rho_{w}}{\rho_{l}}\right)^{1 / 2}
$$

For detailed description of the effect of the pressure and GLR on the predicted two-phase flow pattern see Jedelsky and Jicha (2008). Particular operation points are marked with diamond symbols and connected by a line to group points with constant operational pressure, $p=0.2$ $\mathrm{MPa}$, or GLR $=5 \%$. Levels of the $\overline{G_{n}^{\prime}}$ and $\overline{\dot{m}_{n}^{\prime}}$ values are expressed using a grey scale. It is well seen that the alteration in the flow regime, induced by the GLR change, significantly affects the level of unsteadiness. 
High $\overline{G_{n}^{\prime}}$ and $\overline{\dot{m}_{n}^{\prime}}$ levels are found for $\mathrm{X}>\sim 100$, in the case of the predicted bubbly flow. With increasing GLR (decrease in $X$ coordinate), the two-phase flow changes to frothy and then to the annular pattern, where the unsteadiness levels decrease. The inlet pressure does not lead to an extensive modification of the flow regime and also the unsteadiness levels do not change markedly. In our previous work (Jedelsky and Jicha 2008) we on the same atomizer used a method for the estimation of the flow rate fluctuations based on the measurement of pressure fluctuations inside the mixing chamber of an effervescent atomizer. The flow rate fluctuation levels were high at low GLRs $(\sim 1 \%)$ and decreased with GLR increase, however pressure effect for $0.1 \mathrm{MPa}<p<0.5 \mathrm{MPa}$ was weak. Visualization of the internal two-phase flow shown two processes responsible for a variable void fraction in the nozzle exit as a potential reason for the unsteady spray: large volumes of separated phases and temporally unsteady two-phase flow; both were observed mainly at low GLR. These previous results well agree with overall $\overline{\dot{m}_{n}^{\prime}}$ values (Fig. 16) which implicitly confirms for a direct link between the structure of the internal flow and the resulting spray. It also testifies the consistency of the fundamentally different methods.

\section{CONCLUSIONS}

The combined PLIF-PIV method was used for spatially and temporally resolved measurements of liquid concentration and flux in an effervescent spray.

Time-average radial profiles of the liquid concentration, $\bar{C}$, are bell shaped with their maximum in the nozzle centreline and an approximately exponentially decaying trend at radial distances larger than $10 \mathrm{~mm}$. The $\bar{C}$ profiles vary significantly with GLR and moderately also with the injection pressure. Cumulative radial distributions, $\bar{C}_{c}$, are "s" shaped. Half-angles derived from $\bar{C}_{c}$ profiles show a monotonic, almost linearly decreasing 
tendency with GLR. Effect of the pressure is indistinctive. It is attributed to the internal twophase flow regime and the character of the discharge.

The shape of time-average radial profiles of the mass flux density, $\bar{G}$, and its cumulative value, $\bar{G}_{c}$, generally resembles patterns of their corresponding concentration profiles however the mass flux density profiles are significantly narrower due to the effect of spatial velocity distribution. Also the effect of operation conditions on $\bar{G}$ and $\bar{G}_{c}$ profiles remained the same as for $\bar{C}$ and $\bar{C}_{c}$. Half-angles of temporally averaged fluxes are about one half of the $\bar{C}$ halfangles.

Radial profiles of the temporal fluctuations of the concentration, $\overline{C_{n}^{\prime}}$, and of the flux density, $\overline{G_{n}^{\prime}}$ show a specific pattern with low centreline values and maximum near spray edge. The fluctuations are related to a combined action of two factors; temporally varying GLR at the exit orifice and droplet dynamics. The overall values of these fluctuations were used as a measure of the spray unsteadiness and correlated with internal two-phase flow patterns predicted by a published flow map. The spray unsteadiness is relatively low when operated in the annular flow (high GLR) and increases with the shift to bubbly flow (low GLR) in the published flow map. It confirms a direct link between temporal spray character and internal flow regime.

\section{ACKNOWLEDGEMENT}

The authors greatly acknowledge financial support from project No. 101/11/1264 funded by the Czech Science Foundation.

\section{REFERENCES}

Adrian, R. J. (2011) Particle image velocimetry, New York: Cambridge University Press. 
Aliabadi, A.A., Lim, K.W.J., Rogak, S.N. and Green, S.I. (2011) Steady and transient droplet dispersion in an air-assist internally mixing cone atomizer, Atomization and Sprays, 21(12), pp. 1009-1031.

Process Associates of America (2003) Modified Baker's map for horizontal two-phase flow with transformed coordinates, [online], accessed March 2003, Available at URL: < http://www.processassociates.com/process/fluid/2faz_xy.htm>.

Bachalo, W.D. (1994) The phase Doppler method - analysis, performance evaluations, and applications, Particle \& Particle Systems Characterization, 11(1), pp. 73-83.

Berg, T., Deppe, J., Schucht, T. and Voges, H. (2003) Mass flux imaging in sprays, Proc. of $9^{\text {th }}$ ICLASS, Sorrento, Italy.

Catlin, C.A. and Swithenbank, J. (2001) Physical processes influencing effervescent atomizer performance in the slug and annular flow regimes, Atomization and Sprays, 11(5), pp. 575595.

Chen, S.K. and Lefebvre, A.H. (1994) Spray cone angles of effervescent atomizers, Atomization and Sprays, 4(3), pp. 291-301.

Cohen, J.M. and Rosfjord, T.J. (1991) Spray patternation at high pressure, Journal of Propulsion and Power, 7(4), pp. 481-487.

Daviault, S.G., Ramadan, O.B., Matida, E.A., Hughes, P.M. and Hughes, R. (2012) Atomization performance of petroleum coke and coal water slurries from a twin fluid atomizer, Fuel, 98, pp. 183-193.

Domann, R. and Hardalupas, Y. (2002) Planar droplet sizing for quantification of spray unsteadiness, Proc. of 18th ILASS-Europe, Zaragoza, Spain, paper 090, pp. 1-6, 2002 [on CD-ROM].

Dullenkopf, K., Willmann, M., Wittig, S., Schone, F., Stieglmeier, M., Tropea, C. and Mundo, C. (1998) Comparative mass flux measurements in sprays using a patternator and the 
phase-Doppler technique, Particle \& Particle Systems Characterization, 15(2), pp. 81-89.

Edwards, C.F. and Marx, K.D. (1995) Multipoint statistical structure of the ideal spray. 2. Evaluating steadiness using the interparticle time distribution, Atomization and Sprays, 5(45), pp. 457-505.

Gadgil, H., Dolatabadi, A. and Raghunandan, B.N. (2011) Mass distribution studies in effervescent sprays, Atomization and Sprays, 21(5), pp. 375-390.

Gadgil, H.P. and Raghunandan, B.N. (2011) Some features of spray breakup in effervescent atomizers, Experiments in Fluids, 50(2), pp. 329-338.

Heinlein, J. and Fritsching, U. (2006) Droplet clustering in sprays, Experiments in Fluids, 40(3), pp. 464-472.

Hetsroni, G. and Sokolov, M. (1971) Distribution of mass, velocity, and intensity of turbulence in a 2-phase turbulent jet, Journal of Applied Mechanics, 38(2), pp. 315-327.

Huang, X., Wang, X. and Liao, G. (2008) Visualization of two phase flow inside an effervescent atomizer, Journal of Visualization, 11(4), pp. 299-308.

Jakubik, T., Lawes, M., Woolley, R. and Jicha, M. (2006) Study of ambient turbulence effects on diesel sprays in a fan-stirred vessel, Atomization and Sprays, 16(6), pp. 687-703.

Jedelsky, J. and Jicha, M. (2004) Characteristics of a two-phase flow inside the mixing chamber of an effervescent atomizer, Proc. of 3rd International Symposium on Two-Phase Flow Modelling and Experimentation, Pisa, Italy, pp. 2539-2546.

Jedelsky, J. and Jicha, M. (2008) Unsteadiness in effervescent sprays: A new evaluation method and the influence of operational conditions, Atomization and Sprays, 18(1), pp. 4983.

Jedelsky, J., Jicha, M., Slama, J. and Otahal, J. (2009) Development of an Effervescent Atomizer for Industrial Burners. Energy \& Fuels, 23, pp. 6121-6130.

Koh, H., Jeon, J., Kim, D., Yoon, Y. and Koo, J.Y. (2003) Analysis of signal attenuation for 
quantification of a planar imaging technique, Measurement Science \& Technology, 14(10), pp. $1829-1838$.

Lazaro, B., Peinado, D., Vega, M., Lecuona, A., Rodriguez, P., Jasuja, A. and Liousse, F. (2003) Laser Scattering Patternator: a novel technique for the measurement of industrial, optically dense sprays, Proc. of $9^{\text {th }}$ ICLASS, 8p. Sorrento, Italy.

Leung, J.C. and Epstein, M. (1990) A generalised correlation for 2-phase nonflashing homogeneous choked flow, Journal of Heat Transfer-Transactions of the Asme, 112(2), pp. $528-530$.

Liu, M., Duan, Y.F. and Zhang, T.N. (2010) Evaluation of effervescent atomizer internal design on the spray unsteadiness using a phase/Doppler particle analyzer, Experimental Thermal and Fluid Science, 34(6), pp. 657-665.

Liu, M., Duan, Y.F., Zhang, T.N. and Xu, Y.Q. (2011) Evaluation of unsteadiness in effervescent sprays by analysis of droplet arrival statistics — The influence of fluids properties and atomizer internal design, Experimental Thermal and Fluid Science, 35(1), pp. $190-198$.

Luong, J.T.K. and Sojka, P.E. (1999) Unsteadiness in effervescent sprays, Atomization and Sprays, 9(1), pp. 87-109.

McVey, J.B., Russell, S. and Kennedy, J.B. (1987) High-resolution patternator for the characterisation of fuel sprays, Journal of Propulsion and Power, 3(3), pp. 202-209.

Panchapakesan, N.R. and Lumley, J.L. (1993) Turbulence measurements in axisymmetric jets of air and helium. Part 2. Helium jet, Journal of Fluid Mechanics, 246, pp. 225-247.

Roisman, I.V. and Tropea, C. (2001) Flux measurements in sprays using phase Doppler techniques. Atomization and Sprays, 11(6), pp. 667-699.

Saffman, M. (1987) Automatic calibration of LDA measurement volume size, Applied Optics, 26(13), pp. 2592-2597. 
Sankar, S.V., Maher, K.E. and Robart, D.M. (1999) Rapid characterization of fuel atomizers using an optical patternator, Journal of Engineering for Gas Turbines and PowerTransactions of the Asme, 121(3), pp. 409-414.

Santangelo, P.J. and Sojka, P.E. (1995) A holographic investigation of the near-nozzle structure of an effervescent atomizer-produced spray, Atomization and Sprays, 5(2), pp. 137155.

Sivathanu, Y.R., Lim, J., Wallace, B. and Seei, R. (2010) A comparison of spray angle measurements using optical and mechanical methods, Atomization and Sprays, 20(1), pp. 8592.

Sommerfeld, M. and Qiu, H.H. (1995) Particle concentration measurements by phase-Doppler anemometry in complex dispersed 2-phase flows, Experiments in Fluids, 18(3), pp. 187-198.

Sovani, S.D., Chou, E., Sojka, P.E., Gore, J.P., Eckerle, W.A. and Crofts, J.D. (2001) High pressure effervescent atomization: effect of ambient pressure on spray cone angle, Fuel, 80(3), pp. 427-435.

Sovani, S.D., Crofts, J.D., Sojka, P.E., Gore, J.P. and Eckerle, W.A. (2005) Structure and steady-state spray performance of an effervescent diesel injector, Fuel, 84(12-13), pp. 15031514.

Sutherland, J.J., Sojka, P.E. and Plesniak, M.W. (1997) Ligament Controlled Effervescent Atomization. Atomization and Sprays, 7(4), pp. 383-406.

Whitlow, J.D. and Lefebvre, A.H. (1993) Effervescent Atomizer Operation and Spray Characteristics, Atomization and Sprays, 3(2), pp. 137-155. 


\section{List of Figures}

Fig. 1 Cross section view with basic dimensions of the Plexiglas atomizer (left) and its perspective view (right).

Fig. 2 PIV-PLIF system in stereoscopic PIV arrangement.

Fig. 3 Emission spectra of LHO for excitation wavelength $266 \mathrm{~nm}$ and $355 \mathrm{~nm}$ and fluorescence yield at $416 \mathrm{~nm}$ as a function of the excitation wavelength.

Fig. 4 Lifetime of the fluorescence for IR excitation pulse $7 \mathrm{~ns}$ at $266 \mathrm{~nm}$. The fluorescence decay course is approximated using a two-exponential function model.

Fig. 5 Spatial distribution of $C_{i}(x, y)$ for $p=0.2 \mathrm{MPa}$ and a) GLR $=5 \%$ (left), b) GLR $=2 \%$ (right).

Fig. 6 Typical spatial distribution of the liquid velocity, 3C vector field averaged per 256 shots, $p=0.2 \mathrm{MPa}, \mathrm{GLR}=5 \%$.

Fig. 7 Radial profiles of $\bar{C}$ and $\bar{C}_{c}$ for varying GLR at $p=0.2 \mathrm{MPa}$.

Fig. 8 Radial profiles of $\bar{C}$ and $\bar{C}_{c}$ for varying $p$ at GLR $=5 \%$.

Fig. 9 Radial profiles of $\bar{G}$ and $\bar{G}_{c}$ for varying GLR at $p=0.2 \mathrm{MPa}$.

Fig. 10 Radial profiles of $\bar{G}$ and $\bar{G}_{c}$ for varying $p$ at GLR $=5 \%$.

Fig. 11 Radial profiles of $\overline{C_{n}^{\prime}}$ for varying GLR at $p=0.2 \mathrm{MPa}$.

Fig. 12 Radial profiles of $\overline{C_{n}^{\prime}}$ for varying $p$ at GLR $=5 \%$.

Fig. 13 Radial profiles of $\overline{G_{n}^{\prime}}$ for varying GLR at $p=0.2 \mathrm{MPa}$.

Fig. 14 Radial profiles of $\overline{G_{n}^{\prime}}$ for varying $p$ at GLR $=5 \%$.

Fig. 15 Overall values of $\overline{G_{n}^{\prime}}$ in the two-phase flow map; modified Baker's map for vertical downward flow with transformed coordinates according to Process Associates of America 
(2003) with marked influence of operational conditions on the flow regimes. Arrows show direction of regime change while the appropriate values increase. AB-SP: line defining transition between bubble and plug or annular and slug pattern, A-BF: transition between annular and bubble-froth pattern, A-D: transition between annular and dispersed pattern.

Fig. 16 Overall values of $\overline{\dot{m}_{n}^{\prime}}$ in the two-phase flow map as in Fig. 15. 


\section{List of Tables}

Table 1: Half-angles (degrees) for individual cumulative liquid concentrations for varying GLR at $p=0.2 \mathrm{MPa}$ (left) and for varying $p$ at GLR $=5 \%$ (right).

Table 2: Half-angles (degrees) for individual cumulative liquid fluxes for varying GLR at $p=$ $0.2 \mathrm{MPa}$ (left) and for varying $p$ at GLR $=5 \%$ (right). 
Table 1: Half-angles (degrees) for individual cumulative liquid concentrations for varying GLR at $p=0.2 \mathrm{MPa}$ (left) and for varying $p$ at GLR $=5 \%$ (right).

\begin{tabular}{cccccc}
\hline GLR & $\dot{m}_{g}$ & $\dot{m}_{l}$ & & & \\
$(\%)$ & $(\mathrm{g} / \mathrm{s})$ & $(\mathrm{g} / \mathrm{s})$ & & & \\
\hline 2 & 0.64 & 32 & 5.2 & 7.9 & 11.3 \\
5 & 0.97 & 20 & 5.0 & 7.7 & 10.7 \\
10 & 1.4 & 13 & 4.7 & 7.2 & 10.3 \\
20 & 1.9 & 9.7 & 4.4 & 6.4 & 9.3 \\
50 & 3.1 & 6.3 & 3.9 & 5.6 & 7.9 \\
\hline
\end{tabular}

\begin{tabular}{cccccc}
\hline$p(\mathrm{MPa})$ & $\dot{m}_{g}$ & $\dot{m}_{l}$ & & & \\
& $(\mathrm{~g} / \mathrm{s})$ & $(\mathrm{g} / \mathrm{s})$ & & & \\
\hline 0.1 & 0.69 & 13 & 5.2 & 8.3 & 12.2 \\
0.2 & 0.97 & 20 & 5.0 & 7.7 & 10.7 \\
0.3 & 1.4 & 28 & 5.0 & 7.8 & 11.2 \\
0.4 & 1.7 & 34 & 5.3 & 8.2 & 11.7 \\
0.5 & 2.0 & 42 & 5.3 & 8.2 & 11.6 \\
\hline
\end{tabular}


Table 2: Half-angles (degrees) for individual cumulative liquid fluxes for varying GLR at $p=$ $0.2 \mathrm{MPa}$ (left) and for varying $p$ at GLR $=5 \%$ (right).

\begin{tabular}{cccc}
\hline GLR (\%) & $50 \%$ & $75 \%$ & $90 \%$ \\
\hline 2 & 3.2 & 4.6 & 6.4 \\
5 & 3.1 & 4.5 & 6.2 \\
10 & 2.8 & 4.1 & 5.6 \\
20 & 2.7 & 3.9 & 5.2 \\
50 & 2.5 & 3.6 & 4.6 \\
\hline
\end{tabular}

\begin{tabular}{cccc}
\hline$p(\mathrm{MPa})$ & $50 \%$ & $75 \%$ & $90 \%$ \\
\hline 0.1 & 3.0 & 4.6 & 6.4 \\
0.2 & 3.1 & 4.4 & 6.0 \\
0.3 & 2.9 & 4.3 & 5.7 \\
0.4 & 3.1 & 4.6 & 6.2 \\
0.5 & 3.3 & 4.7 & 6.2 \\
\hline
\end{tabular}



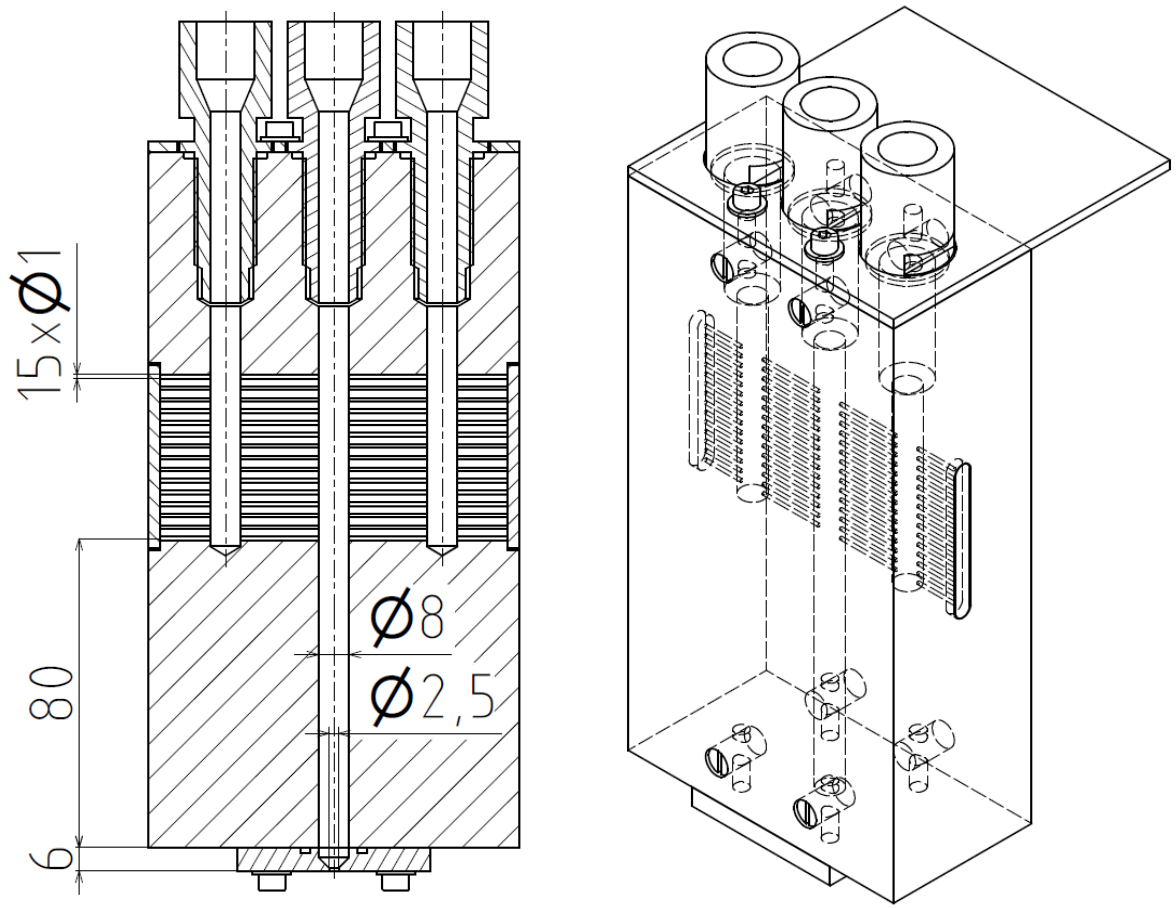

Fig. 1 Cross section view with basic dimensions of the Plexiglas atomizer (left) and its perspective view (right). 


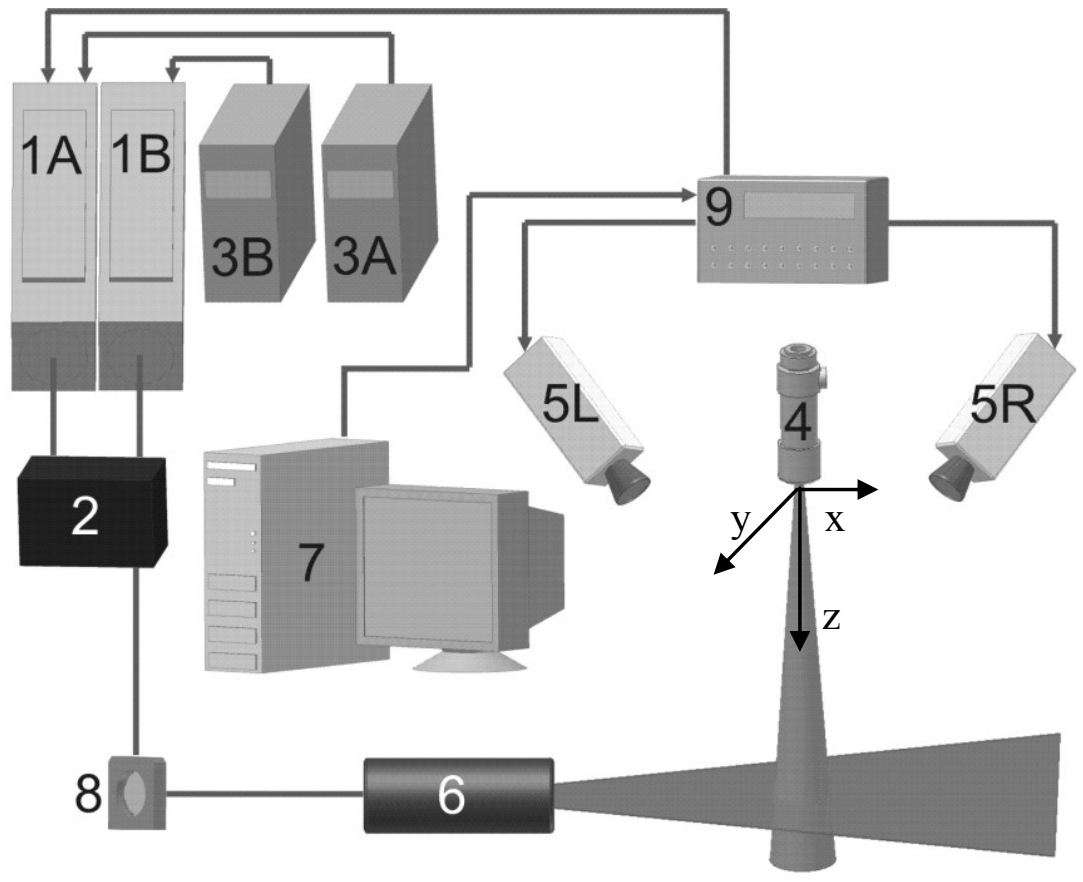

Fig. 2 PIV-PLIF system in stereoscopic PIV arrangement. 


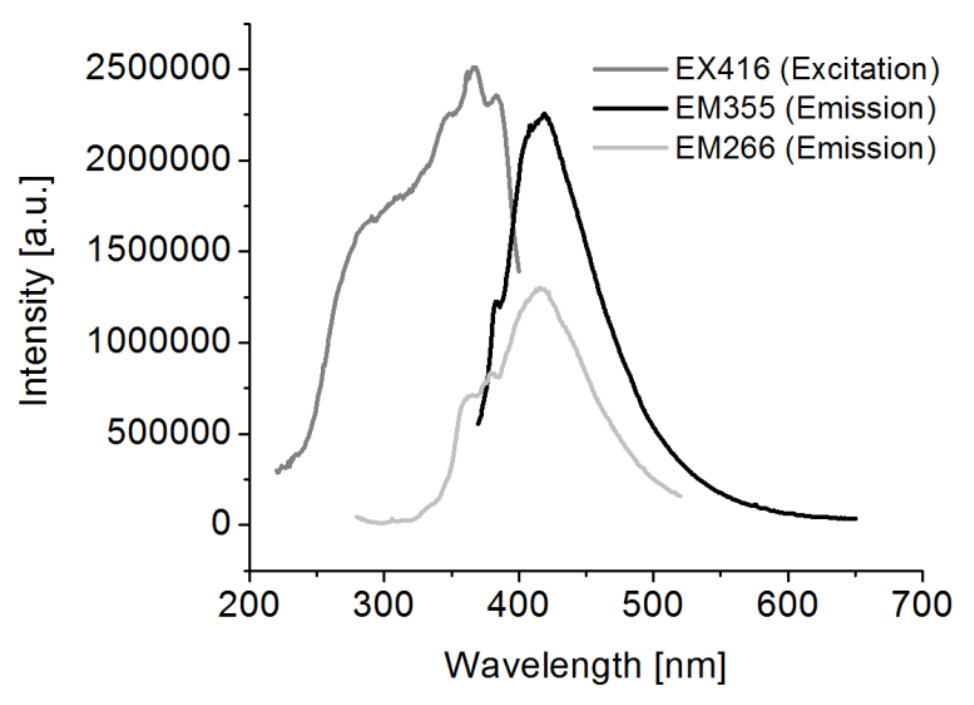

Fig. 3 Emission spectra of LHO for excitation wavelength $266 \mathrm{~nm}$ and $355 \mathrm{~nm}$ and fluorescence yield at $416 \mathrm{~nm}$ as a function of the excitation wavelength. 


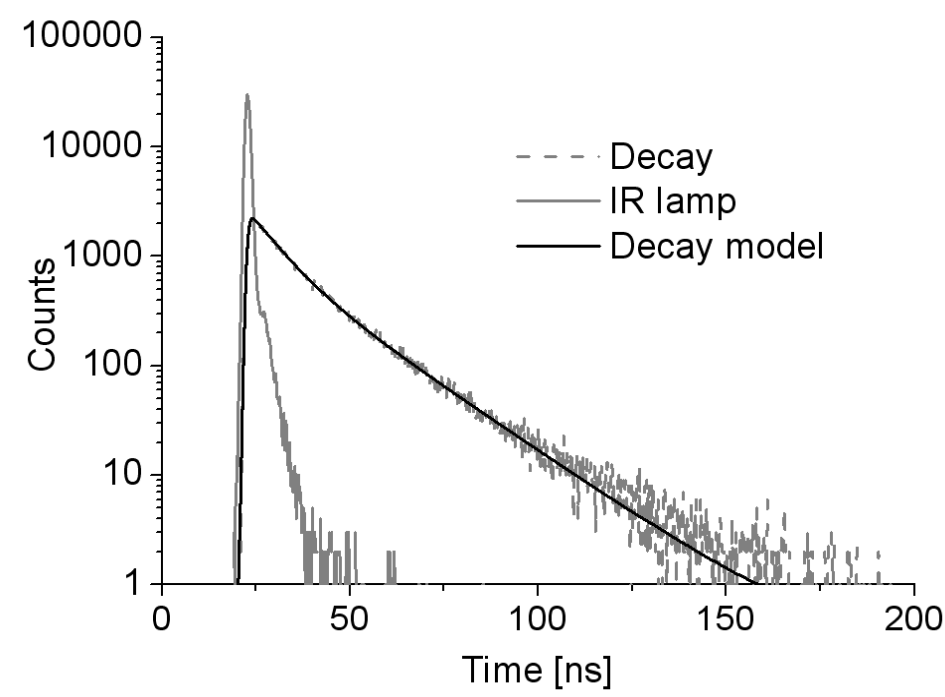

Fig. 4 Lifetime of the fluorescence for IR excitation pulse $7 \mathrm{~ns}$ at $266 \mathrm{~nm}$. The fluorescence decay course is approximated using a twoexponential function model. 

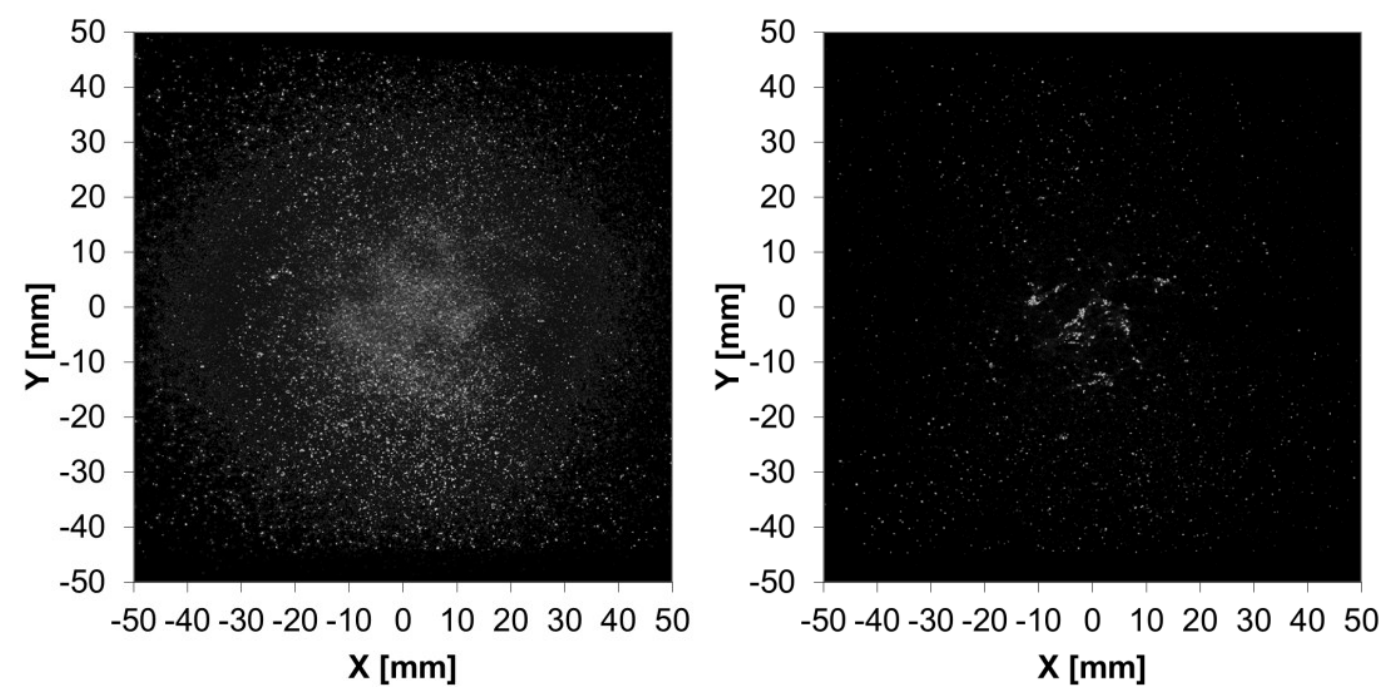

Fig. 5 Spatial distribution of $C_{i}(x, y)$ for $p=0.2 \mathrm{MPa}$ and a) GLR $=5 \%$ (left), b) GLR $=2 \%$ (right) 


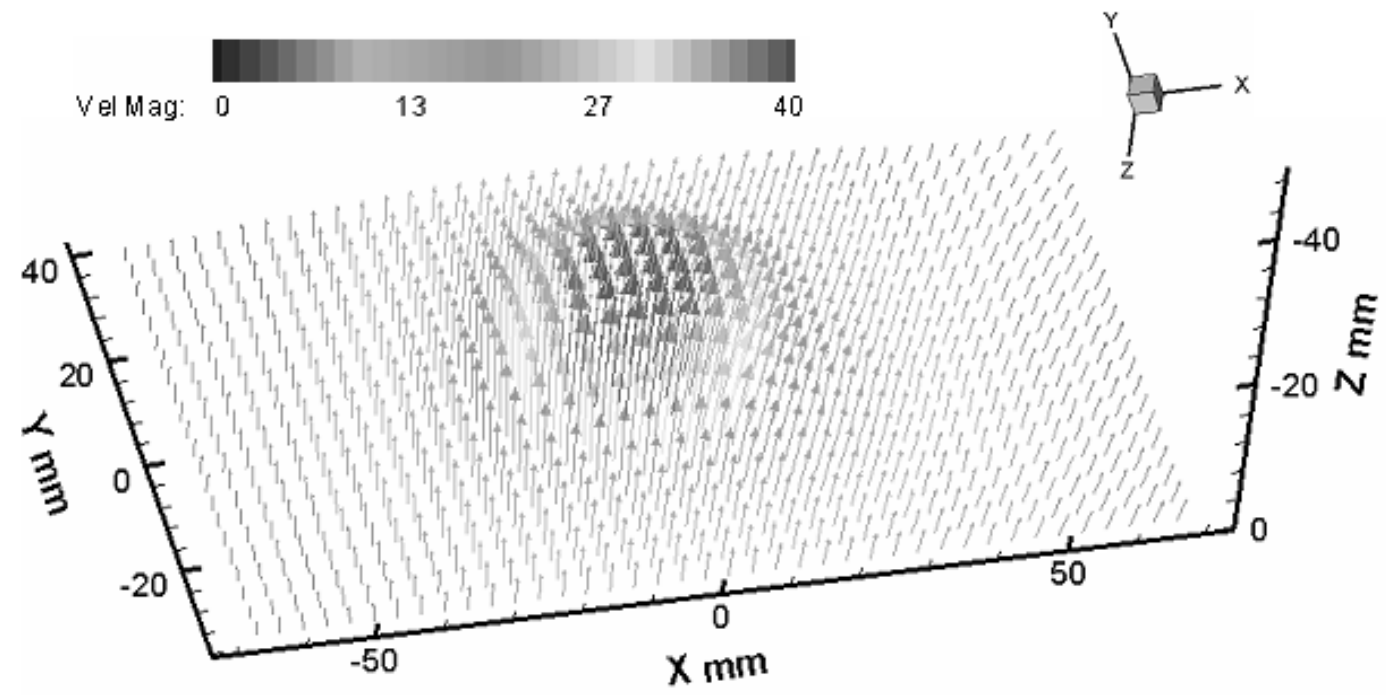

Fig. 6 Typical spatial distribution of the liquid velocity, 3C vector field averaged per 256 shots, $p=0.2 \mathrm{MPa}, \mathrm{GLR}=5 \%$. 


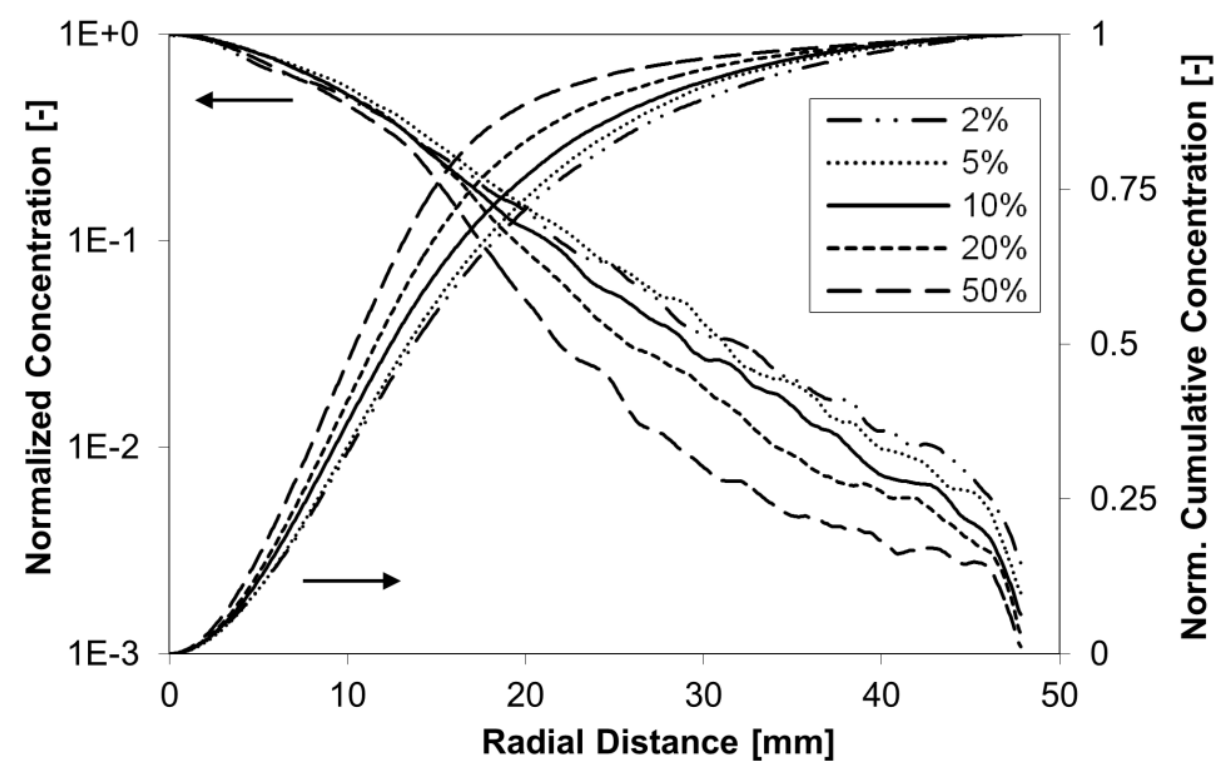

Fig. 7 Radial profiles of $\bar{C}$ and $\bar{C}_{c}$ for varying GLR at $p=0.2 \mathrm{MPa}$. 


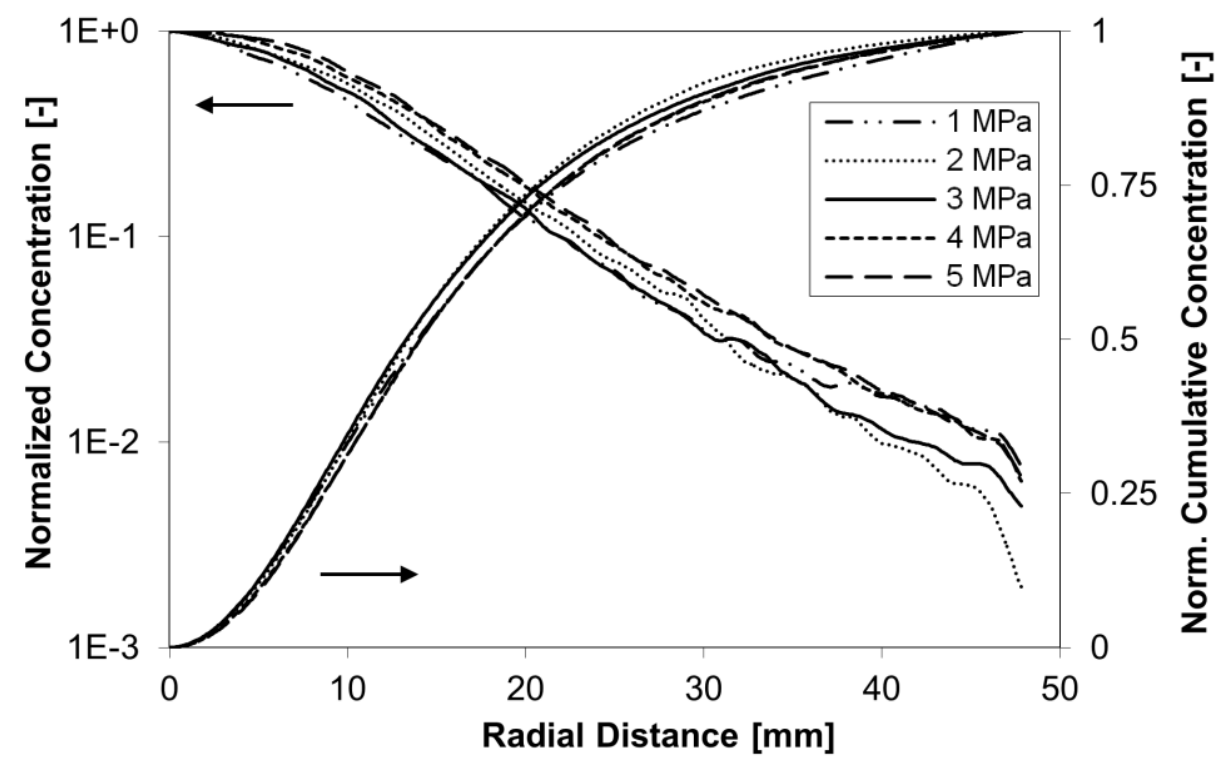

Fig. 8 Radial profiles of $\bar{C}$ and $\bar{C}_{c}$ for varying $p$ at GLR $=5 \%$. 


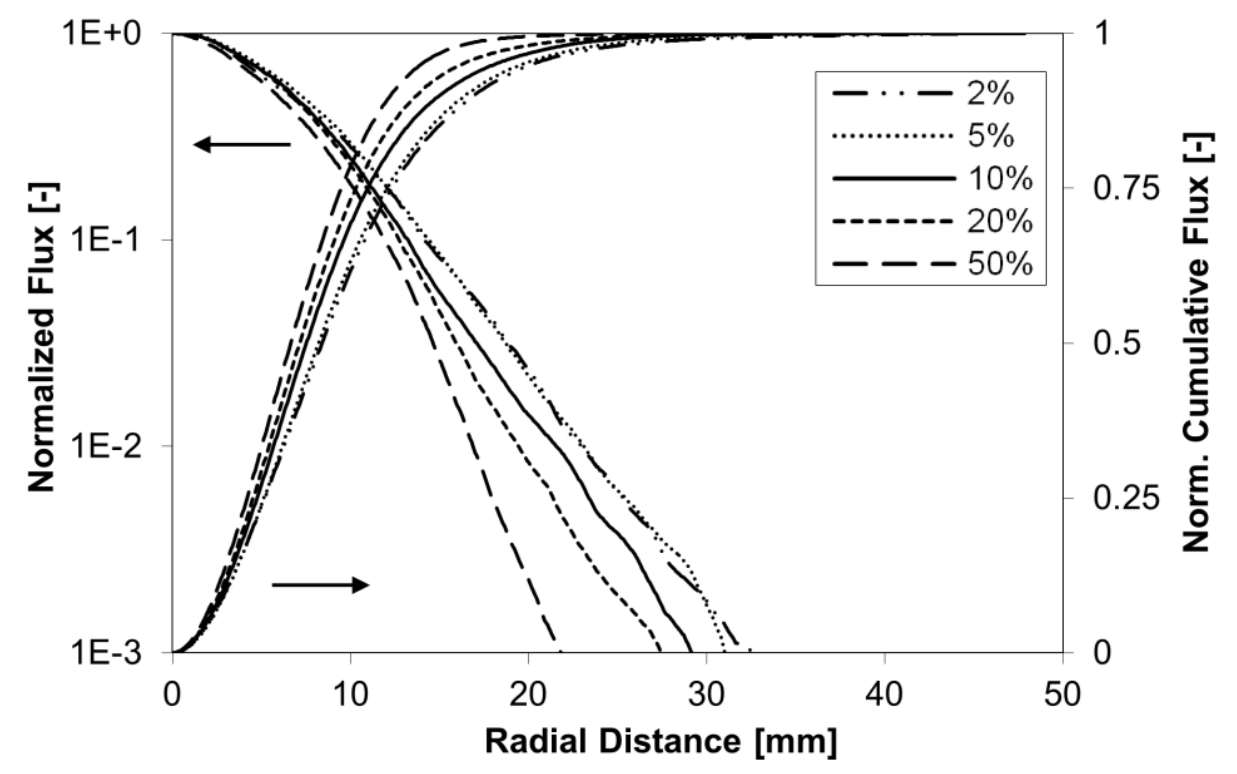

Fig. 9 Radial profiles of $\bar{G}$ and $\bar{G}_{c}$ for varying GLR at $p=0.2 \mathrm{MPa}$. 


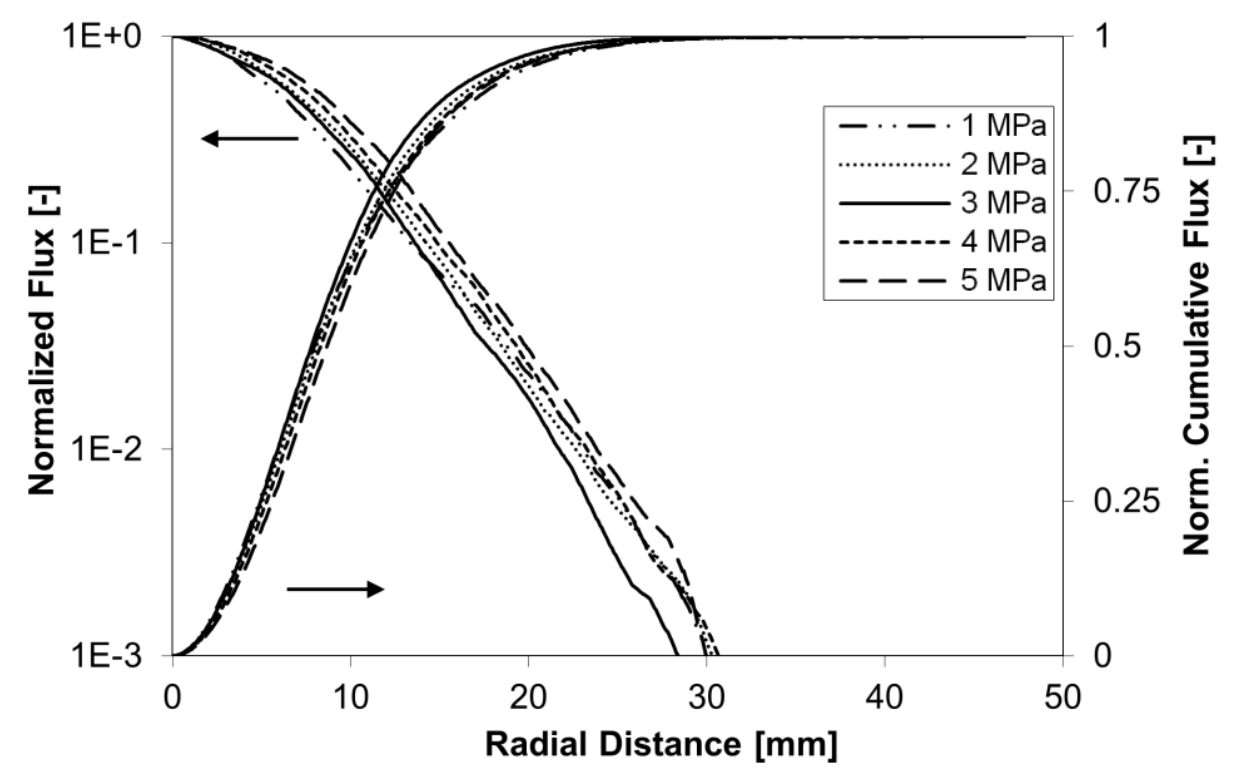

Fig. 10 Radial profiles of $\bar{G}$ and $\bar{G}_{c}$ for varying $p$ at GLR $=5 \%$. 


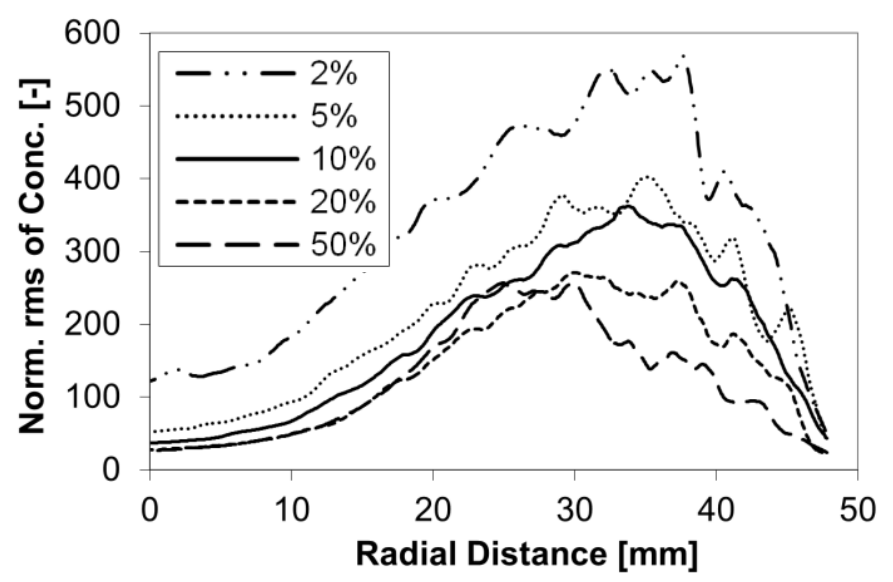

Fig. 11 Radial profiles of $\overline{C_{n}^{\prime}}$ for varying GLR at $p=0.2 \mathrm{MPa}$. 


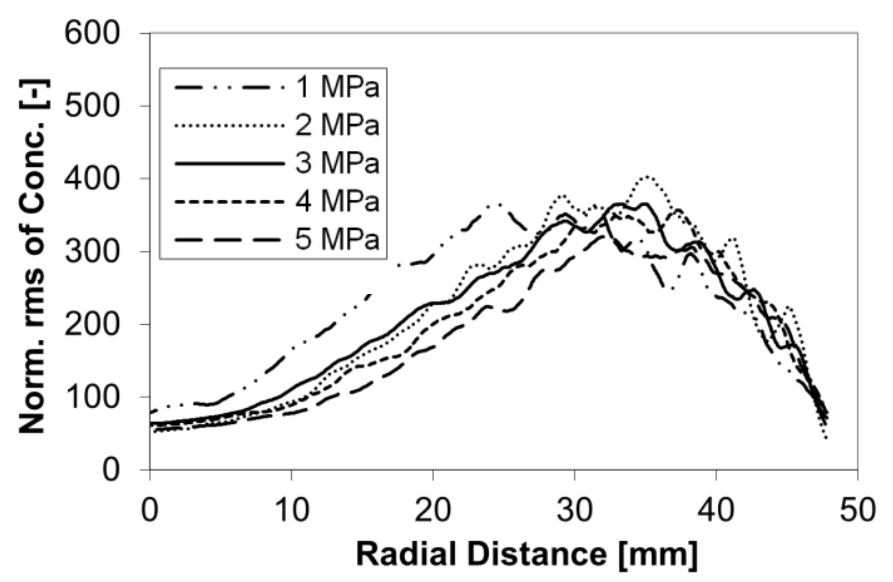

Fig. 12 Radial profiles of $\overline{C_{n}^{\prime}}$ for varying $p$ at GLR $=5 \%$. 


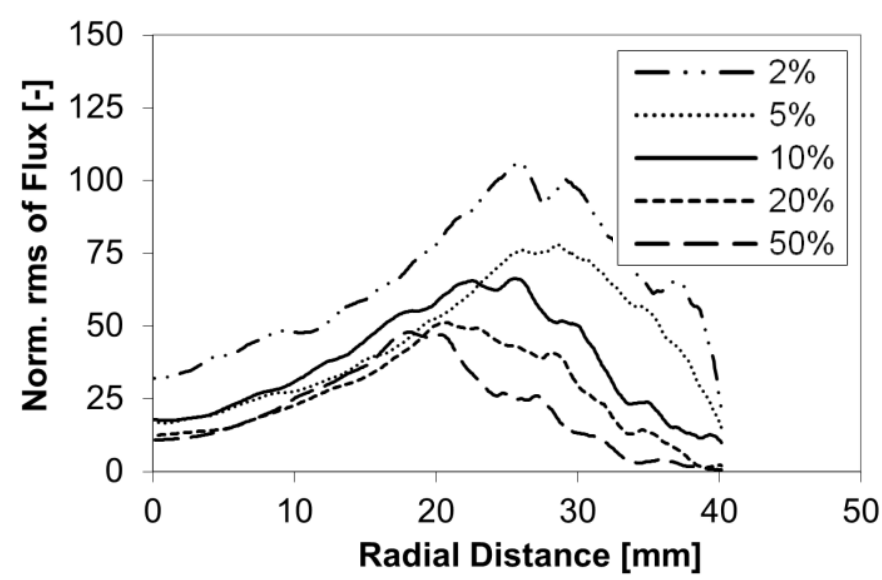

Fig. 13 Radial profiles of $\overline{G_{n}^{\prime}}$ for varying GLR at $p=0.2 \mathrm{MPa}$. 


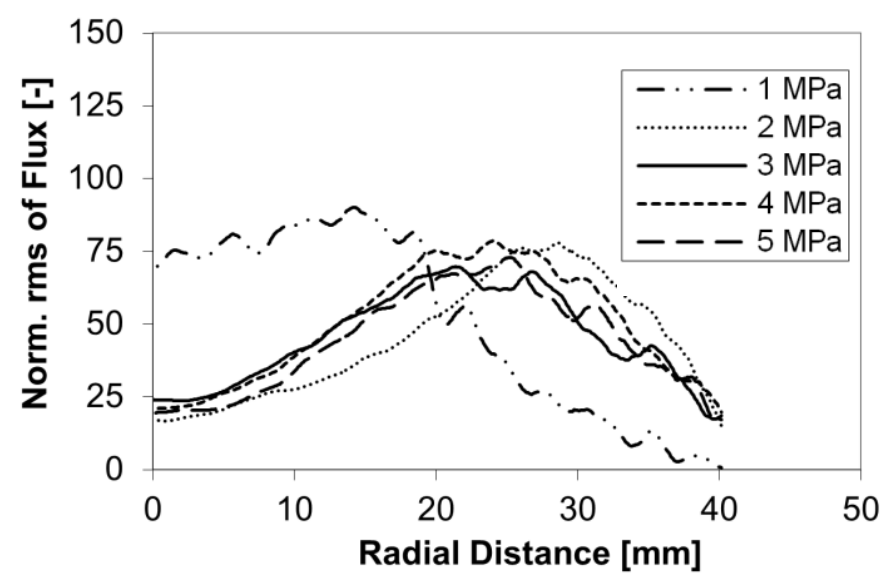

Fig. 14 Radial profiles of $\overline{G_{n}^{\prime}}$ for varying $p$ at GLR $=5 \%$. 


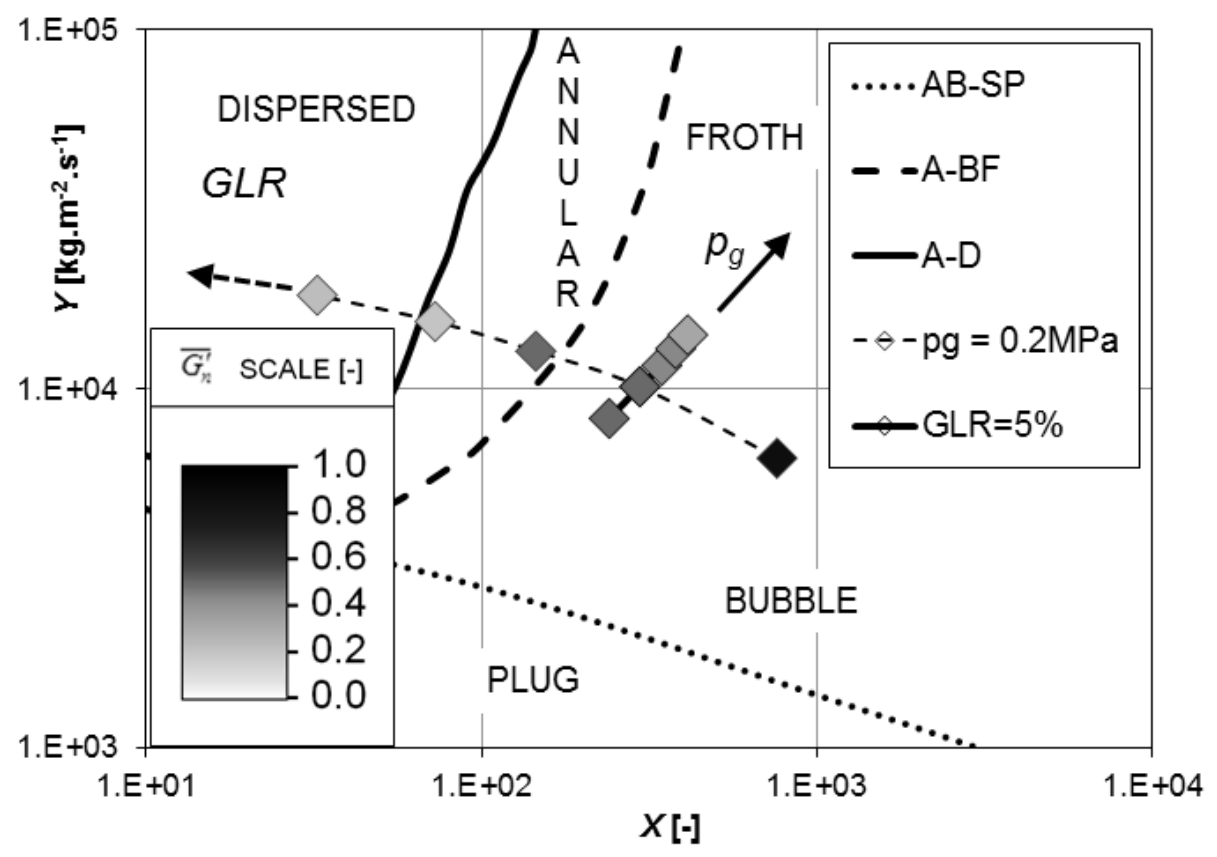

Fig. 15 Overall values of $\overline{G_{n}^{\prime}}$ in the two-phase flow map; modified Baker's map for vertical downward flow with transformed coordinates according to Process Associates of America (2003) with marked influence of operational conditions on the flow regimes. Arrows show direction of regime change while the appropriate values increase. AB-SP: line defining transition between bubble and plug or annular and slug pattern, A-BF: transition between annular and bubble-froth pattern, A-D: transition between annular and dispersed pattern. 


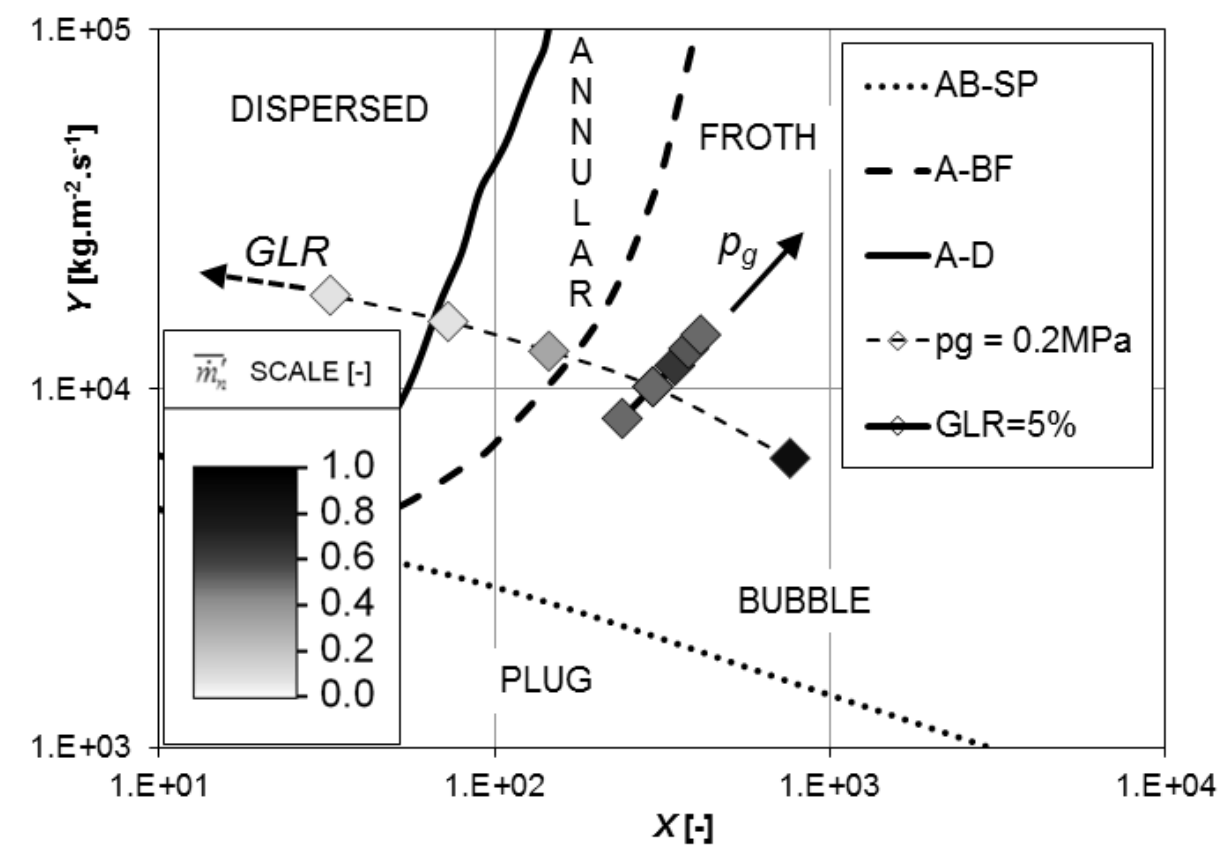

Fig. 16 Overall values of $\overline{\dot{m}_{n}^{\prime}}$ in the two-phase flow map as in Fig. 15. 